\title{
BOW SHOCK FRAGMENTATION DRIVEN BY A THERMAL INSTABILITY IN LABORATORY ASTROPHYSICS EXPERIMENTS
}

\author{
F. Suzuki-Vidal ${ }^{1}$, S. V. Lebedev ${ }^{1}$, A. Ciardi ${ }^{2,3}$, L. A. Pickworth ${ }^{1,7}$, R. Rodriguez ${ }^{4}$, J. M. Gil ${ }^{4}$, G. Espinosa ${ }^{4}$, P. Hartigan ${ }^{5}$, \\ G. F. Swadling ${ }^{1,7}$, J. Skidmore ${ }^{1,8}$, G. N. Hall ${ }^{1,7}$, M. Bennett ${ }^{1}$, S. N. Bland ${ }^{1}$, G. BurdiaK ${ }^{1}$, P. De Grouchy ${ }^{1,9}$, J. Music ${ }^{1}$, \\ L. Suttle ${ }^{1}$, E. Hansen ${ }^{6}$, And A. Frank ${ }^{6}$ \\ ${ }^{1}$ Blackett Laboratory, Imperial College London, Prince Consort Road, London SW7 2BW, UK; f.suzuki@imperial.ac.uk \\ ${ }^{2}$ Sorbonne Universités, UPMC Univ. Paris 6, UMR 8112, LERMA, F-75005, Paris, France \\ ${ }^{3}$ LERMA, Observatoire de Paris, PSL Research University, CNRS, UMR 8112, F-75014, Paris, France \\ ${ }^{4}$ Departamento de Fisica de la Universidad de Las Palmas de Gran Canaria, E-35017 Las Palmas de Gran Canaria, Spain \\ 5 Department of Physics and Astronomy, Rice University, 6100 S. Main, Houston, TX 77521-1892, USA \\ ${ }^{6}$ Department of Physics and Astronomy, University of Rochester, Rochester, NY 14627, USA \\ Received 2015 September 14; accepted 2015 November 5; published 2015 December 14
}

\begin{abstract}
The role of radiative cooling during the evolution of a bow shock was studied in laboratory-astrophysics experiments that are scalable to bow shocks present in jets from young stellar objects. The laboratory bow shock is formed during the collision of two counterstreaming, supersonic plasma jets produced by an opposing pair of radial foil Z-pinches driven by the current pulse from the MAGPIE pulsed-power generator. The jets have different flow velocities in the laboratory frame, and the experiments are driven over many times the characteristic cooling timescale. The initially smooth bow shock rapidly develops small-scale nonuniformities over temporal and spatial scales that are consistent with a thermal instability triggered by strong radiative cooling in the shock. The growth of these perturbations eventually results in a global fragmentation of the bow shock front. The formation of a thermal instability is supported by analysis of the plasma cooling function calculated for the experimental conditions with the radiative packages ABAKO/RAPCAL.
\end{abstract}

Key words: Herbig-Haro objects - instabilities - ISM: jets and outflows - methods: laboratory: atomic - plasmas shock waves

\section{INTRODUCTION}

One of the characteristic features of protostellar jets is the presence of shocks. They can be seen as large-scale terminal bow shocks or working surfaces (known as Herbig-Haro or $\mathrm{HH}$ objects) that form as the jet interacts with previous jet ejections. Smaller-scale, internal shocks are also present, which are driven by highly variable flow velocity in the jet. These are formed as the flow moves away from the protostar and encounters and overtakes slower material from previous ejections, with the process repeating along the jet beam. Proper-motion measurements of the jets in HH 34 (Reipurth et al. 2002) and HH 111 (Hartigan et al. 2001) show that the jet flow can reach peak velocities of $\sim 200-300 \mathrm{~km} \mathrm{~s}^{-1}$ with a typical velocity variation along the jet of $\sim 40 \mathrm{~km} \mathrm{~s}^{-1}$.

Shocks from protostellar jets exhibit complex dynamics in which different effects such as shear, hydrodynamic instabilities, and radiative cooling can be present simultaneously (Hartigan 2003). In this work we are particularly interested in the effect of radiative cooling, as it can drastically modify the shock morphology. Radiative losses are strongly dependent on the opacity (Drake et al. 2006), and if the shock region is optically thin, then radiation can escape the shock, leading to an increase in the postshock density. As the shock cools down, it can be prone to the growth of thermal instabilities (Field 1965; Hunter 1970), which can fragment and ultimately break up the shock. This effect has previously been studied mostly through numerical simulations (see, e.g., Blondin

\footnotetext{
${ }^{7}$ Present address: Lawrence Livermore National Laboratories, CA, USA.

${ }^{8}$ Present address: AWE Aldermaston, UK.

${ }^{9}$ Present address: Cornell University, NY, USA.
}

et al. 1989, 1990; de Gouveia dal Pino \& Benz 1993; Stone \& Norman 1993; Frank et al. 1998; Teşileanu et al. 2008 and Asahina et al. 2014).

In this paper we describe laboratory experiments that provide a complementary approach to study the effects of radiative cooling on the structure of the bow shocks. The similarity in the key dimensionless parameters characterizing our experiments, such as the Mach number and the cooling parameter, mean that the results are scalable to the internal shocks observed in young stellar object (YSO) jets. We observe the formation of a bow shock in the flow and its subsequent fragmentation, consistent with the onset of thermal instabilities. The bow shock in the experiments is formed from the interaction of two counterstreaming flows, which is equivalent to observations of internal shocks in YSO jets from a reference frame moving with the shock. An overall approach to modeling astrophysical phenomena in laboratory experiments is presented in the review by Remington et al. (2006), while a recent review of the synergy between observations, theory, and experiments relevant to the studies of YSO jets can be found in Frank et al. (2014).

\section{EXPERIMENTAL SETUP}

The experimental setup is represented schematically in Figure 1. Two counterstreaming, supersonic plasma outflows are produced using two coaxial and oppositely facing radial foil Z-pinches (Suzuki-Vidal et al. 2009). Each radial foil is a metallic disk ( $40 \mathrm{~mm}$ diameter, $14 \mu \mathrm{m}$ thick aluminum), subjected to a fast-rising electrical current pulse from the MAGPIE generator (Mitchell et al. 1996), which for the present experiments used a peak current of $\sim 1 \mathrm{MA}$ in $\sim 330 \mathrm{~ns}$. The 

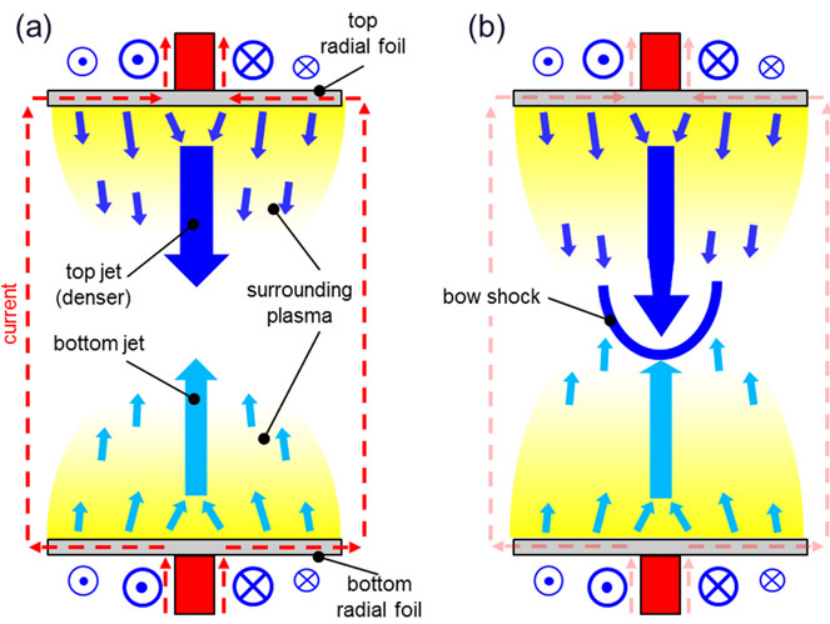

Figure 1. Schematic experimental configuration to study the formation of a bow shock from the interaction between two counterstreaming jets with different relative axial velocities, represented as opposite vertical arrows on axis. The schematic depicts a side-on (radial), cut view of the system, which has azimuthal symmetry. The dashed (red) arrows represent the path of the current that drives the two plasma flows. The (blue) arrows pointing into and out of the page correspond to the azimuthal magnetic field generated by the current, which provides the driving force for the two outflows. The jets are surrounded by lower-density plasma (yellow regions), which moves with the same axial velocity as the jets. Smaller arrows in these regions represent the plasma flow direction. The images depict the two counterstreaming outflows (a) before their collision and (b) after they collide, triggering the formation of a bow shock moving toward the bottom foil.

current is driven to the foils through $6.35 \mathrm{~mm}$ diameter stainless steel tubes touching each foil at its center, with the same current going through both of the foils via vertical posts (shown schematically in Figure 1(a)). The distance between the two foil surfaces was $\sim 30 \mathrm{~mm}$

The plasma is produced by continuous ablation of the surfaces of the foils as they are heated by the current, and the ablated plasma is accelerated by the axial pressure gradient produced by the current-induced azimuthal magnetic field as it diffuses through the foils. Each of the foils produces a supersonic plasma outflow that consists of a dense central jet surrounded by lower-density ambient plasma. Both components propagate with the same axial velocity of $\sim 50-100 \mathrm{~km} \mathrm{~s}^{-1}$. Details on the formation of the outflows by a single radial foil Z-pinch can be found in Suzuki-Vidal et al. (2009), Ciardi et al. (2009), Gourdain et al. (2010), and SuzukiVidal et al. (2012). In the experiments presented here, the interaction of the jets was diagnosed from the side-on (radial) direction using an optical framing camera (Invisible Vision UHSi 12/24) that imaged the optical self-emission from the plasma. This camera is capable of taking up to 12 images per experiment, with $5 \mathrm{~ns}$ exposure and $30 \mathrm{~ns}$ interframe separation. We also used simultaneous optical laser shadowgraphy and interferometry ( $\lambda=532 \mathrm{~nm}$, pulse duration $0.3 \mathrm{~ns})$. The latter diagnostic was used to measure the electron density distribution of the different plasma features present, i.e., jets, surrounding plasma, and bow shock (Swadling et al. 2014).

\subsection{Scaling}

Jets from YSOs are generally well described by ideal magnetohydrodynamics (MHD), and the experiments presented here are designed to produce flows in a similar regime. It is evident that these evolve over hugely different length scales and timescales and have different physical characteristics in terms of density, temperature, and chemical composition. Nevertheless, the invariance properties of the ideal MHD equations provide a framework that allows meaningful scaling of the jet dynamics over many orders of magnitude (see, e.g., Ryutov et al. 1999, 2000, 2001), provided that certain constraints between the flow variables are satisfied. It is important to note that such invariance is also applicable to flows with shocks and to radiative flows under a more restrictive set of constraints (Falize et al. 2011).

In order for a fluid description to be applicable, we require the flows to have a localization parameter $\delta \ll 1$. This is equivalent to an ion mean free path much less than the characteristic spatial scale of the system. Using the parameters from Table 1 in Hartigan et al. (2009), we estimate that jets from YSOs have $\delta \sim 10^{-5}$. Applicability of an MHD description also requires that the transport of momentum, magnetic field, and thermal energy occurs predominantly through advection with the flow, i.e., negligible dissipation through viscosity (Reynolds number $\operatorname{Re} \gg 1$ ), magnetic diffusivity (magnetic Reynolds number $\operatorname{Re}_{M} \gg 1$ ), and heat conduction (Peclet number $\mathrm{Pe} \gg 1$ ), respectively. Owing to their large spatial scales, YSO jets are characterized by Re, $\operatorname{Re}_{M}, \mathrm{Pe} \gtrsim 10^{7}$. Our experiments are characterized by $\delta \sim 10^{-4}$, $\operatorname{Re} \sim 10^{5}, \mathrm{Pe} \sim 10^{3}$, and $\operatorname{Re}_{M} \sim 10^{3}$ (Suzuki-Vidal et al. 2012), and thus we expect a similar overall physical behavior.

Additionally, the interaction of radiative jets with the interstellar medium can be broadly classified by three dimensionless parameters (Blondin et al. 1990): the Mach number $M$ (the ratio of flow speed to sound speed), the density contrast $\eta$ (the ratio of density between the flow and the ambient medium where it propagates), and the cooling parameter $\chi_{\text {cool }}$ (the ratio of the cooling time $\tau_{\text {cool }}$ to the characteristic hydrodynamical time of the flow $\tau_{\text {hydro }}$ ), which quantifies the effect of radiative losses in the plasma. The specific values of $M, \eta$, and $\chi_{\text {cool }}$ essentially determine the overall morphology of the flow. Internal bow shocks in YSO jets are characterized by a relative velocity of $\sim 40 \mathrm{~km} \mathrm{~s}^{-1}$ and $M \sim 10$, and we can expect $\eta \sim 1$ and $\chi_{\text {cool }} \lesssim 1$ (Hartigan et al. 2009). As will be discussed later in the paper, the values of these three parameters in our experiments are close to those in YSO jets.

Overall, the similarity of the dimensionless parameters allows applying the Eulerian scaling relations described in detail in Ryutov et al. (1999, 2000, 2001). Flows with identical Mach numbers will evolve with identical morphology, but on different hydrodynamic temporal $\left(\tau_{\text {hydro }}\right)$ and spatial $\left(r_{\text {jet }}\right)$ scales, related via the corresponding flow velocities $\left(V_{\text {flow }}\right)$ as $\tau_{\text {hydro }}=r_{\text {jet }} / V_{\text {flow }}$. Taking the jet radius as a characteristic spatial scale, young stellar jets typically have $r_{\text {YSO }} \sim 50 \mathrm{AU} \sim 10^{15} \mathrm{~mm}$ and flow velocities of $V_{\text {YSO }}=40 \mathrm{~km} \mathrm{~s}^{-1}$ (i.e., typical velocity variability in YSO jets). For the experiments here $r_{\exp } \sim 1.5 \mathrm{~mm}$ and $V_{\exp }=140 \mathrm{~km} \mathrm{~s}^{-1}$ (i.e., the relative velocity of a single jet in our experiments; see explanation in the next section). Thus, the characteristic temporal scales are $\tau_{\text {YSO }}=1.9 \times 10^{8} \mathrm{~s}(\sim 6 \mathrm{yr})$ and $\tau_{\exp }=10 \mathrm{~ns}$, respectively. The total time interval over which the evolution of the flows is followed in the experiments of $\sim 300 \mathrm{~ns} \quad\left(\approx 30 \tau_{\text {exp }}\right)$ thus corresponds to $\sim 180 \mathrm{yr}$ of evolution for the astrophysical counterpart, which exceeds the typical timescale for multi-epoch observations of YSOs with 

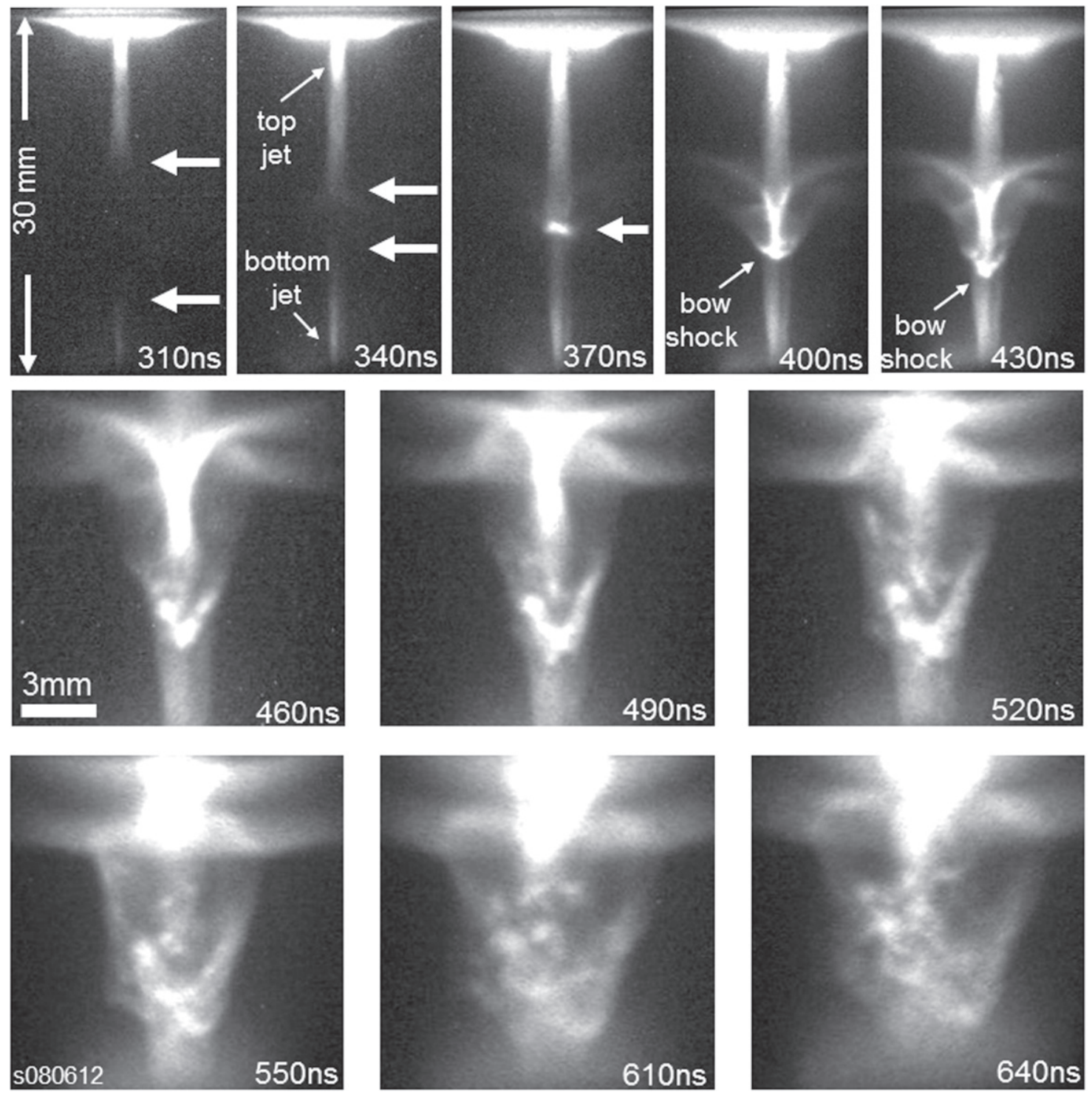

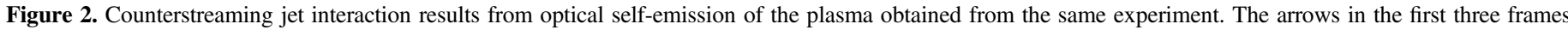

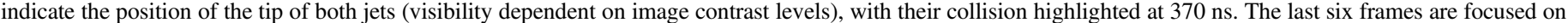
the bow shock region, which is seen to fragment most evidently in the last three times.

the Hubble Space Telescope (HST) of $\sim 10 \mathrm{yr}$ (see, e.g., Hartigan et al. 2011).

\section{EXPERIMENTAL RESULTS AND DISCUSSION}

The overall evolution of the interaction of the two counterstreaming plasma jets can be seen in Figure 2, which shows side-on optical emission images obtained in the same experiment at different times after the start of the current pulse driving the jets. Images in the top row of Figure 2 show the entire region between the two foils and correspond to the early times of the interaction (310-430 ns). The images show the formation of two well-collimated jets on the axis of the system propagating toward each other. Although the top and bottom foils are nominally the same and are driven by the same current, an asymmetry between the top and bottom flows is evident from the emission images. The top jet is formed first compared to the bottom jet, which is seen by looking at the positions of the tips of the jets indicated by horizontal arrows on the first three frames. This asymmetry is caused by opposite- polarity current drive for each foil (radially outward on the bottom foil compared to radially inward on the top foil) and is reproducible from experiment to experiment. The asymmetry affects the details of the plasma ablation and initial acceleration of the flow at the foil (Gourdain \& Seyler 2013). This results in different flow parameters (e.g., ram pressures), which, as the jet collide, lead to the formation of a bow shock. This is first evidenced at $370 \mathrm{~ns}$ as a highly emitting region aligned with the head-on collision between the two jet tips. The bow shock is fully formed at $400 \mathrm{~ns}$ and is seen to move downward, i.e., toward the bottom foil. At this time the postshock region is seen as a highly compressed, highly collimated column, while the bow shock begins to develop small-scale structures from here onward. The long-term evolution of the bow shock and of these structures is highlighted in the subsequent images, corresponding to times 460-640 ns.

The first collision between the two jets seen in the image at 370 ns occurs $\sim 2 \mathrm{~mm}$ below the midplane, which suggests that the two jets have slightly different velocities. This is confirmed 
by following the positions of the visible tips of the jets in time before their collision, resulting in tip velocities of $V_{\text {top jet }} \sim 80 \pm 10 \mathrm{~km} \mathrm{~s}^{-1}$ and $V_{\text {bottom jet }} \sim 60 \pm 10 \mathrm{~km} \mathrm{~s}^{-1}$. The higher velocity in the top jet, combined with a larger density inferred from the stronger self-emission and from laser interferometry, implies an imbalanced ram pressure $\left(P_{\text {ram }}=\rho V^{2}\right)$ in the collision, which explains the shape and orientation of the bow shock and its downward propagation. The velocity of the leading edge of the bow shock was measured as $V_{\text {bow }} \sim 40 \pm 10 \mathrm{~km} \mathrm{~s}^{-1}$.

The velocity of the bow shock measured in the experiments can be compared with the velocity expected from a onedimensional, momentum flux conservation argument (Norman et al. 1983; Hartigan 1989; Blondin et al. 1990; de Gouveia dal Pino \& Benz 1994; de Gouveia Dal Pino 2005; Nicolaï et al. 2008). This is convenient to do in the reference frame of a single jet driving a shock through a stationary medium ahead of it. The velocity of a bow shock $V_{\text {bow }}$ is related to the velocity of the jet $V_{\text {jet }}$ and the density contrast $\eta$ between the jet and the preshock external medium by $V_{\text {bow }} \approx V_{\text {jet }}\left(1+\eta^{-1 / 2}\right)^{-1}$. The measured experimental jet tip velocities of $V_{\text {jet top }} \sim 80 \mathrm{~km} \mathrm{~s}^{-1}$ and $V_{\text {jet bottom }} \sim 60 \mathrm{~km} \mathrm{~s}^{-1}$ for the top and bottom jets, respectively, can be translated into a single jet with a relative tip velocity of $V_{\text {jet rel }}=140 \mathrm{~km} \mathrm{~s}^{-1}$, which in turns forms a bow shock with a relative velocity of $V_{\text {bow rel }}=100 \mathrm{~km} \mathrm{~s}^{-1}$. Using these relative velocities in the equation above results in a density contrast $\eta \sim 6$, i.e., the experiment is equivalent to the interaction of a single jet that is six times denser than the ambient medium where the shock is generated; for this particular counterstreaming geometry, the ambient medium is the opposite (bottom) jet. We can calculate the internal Mach number of the jet working surface by taking the ratio of the jet relative velocity $\left(V_{\text {jet rel }}=140 \mathrm{~km} \mathrm{~s}^{-1}\right)$ and the typical ion acoustic speed in the jet flow of $c_{\mathrm{s}} \sim 15 \mathrm{~km} \mathrm{~s}^{-1}$ (Suzuki-Vidal et al. 2012), resulting in an internal Mach number of $M \sim 10$. Therefore, the values of $\eta$ and $M$ in the experiments are similar to those in YSO jets (Hartigan et al. 2009), and thus the experiments should evolve with a similar jet/shock morphology as discussed earlier.

In addition to the bow shock, the images in Figure 2 also show the collision between the two flows off-axis, i.e., the plasma surrounding the jets, which leads to the formation of a double-shock structure that extends at large radii. This feature is especially evident at the top of the images from $\sim 460 \mathrm{~ns}$ onward. These standing shocks remain approximately symmetric with respect to the midplane between the two foils. Preliminary MHD simulations indicate that these features arise as a result of the presence of toroidal magnetic field advected by the counterstreaming flows (Suzuki-Vidal et al. 2014). A more detailed discussion of the pileup of the magnetic fields will be published separately.

\subsection{Bow Shock and Jet Working Surface: Evolution and Small-scale Structures}

The emission images in Figure 2 show in detail the evolution of the bow shock from its formation at $400 \mathrm{~ns}$ through its rapid fragmentation. At $400 \mathrm{~ns}$ the surface of the bow shock and the postshock region just behind it are smooth. The formation of small-scale structures occurs on timescales that are shorter than the interframe separation for this particular diagnostic. This gives an upper limit for their development timescale of $\sim 30 \mathrm{~ns}$, which is consistent with the appearance of new structures between frames at later times. The characteristic spatial scale of the structures seen in the images ranges from $\sim 200 \mu \mathrm{m}$ to $\sim 2 \mathrm{~mm}$, and at the final stages of the evolution (the last two panels at 610 and $640 \mathrm{~ns}$ ) the bow shock has fragmented into a large number of emitting clumps. The smallest observed size $(\sim 200 \mu \mathrm{m})$ seen on the images obtained with the optical framing camera is comparable to the spatial resolution of this diagnostic and is also comparable to the motional blurring due to the temporal resolution of the diagnostic $\left(5 \mathrm{~ns} \times 40 \mathrm{~km} \mathrm{~s}^{-1}=200 \mu \mathrm{m}\right)$.

Further details of the small-scale structures present in the bow shock and postshock region were obtained with laser probing (0.3 ns pulse duration), which has significantly better spatial resolution $(\lesssim 50 \mu \mathrm{m})$ and reduced motional blurring $(\sim 10 \mu \mathrm{m})$. The interferometry channel of this diagnostic provides measurements of the spatial distribution of the plasma electron density, and the shadowgraphy channel, which is sensitive to spatial gradients of the electron density, provides information on the characteristic spatial scales of the nonuniformities. Figure 3 shows laser probing images obtained at $400 \mathrm{~ns}$ in the same experiment as the optical emission images shown in Figure 2, i.e., the timing of the laser probing images corresponds to the fourth panel in Figure 2. Figure 3(a) shows the raw image from the interferometry channel. The apparent distortion of the interference fringes, which were initially horizontal and uniformly spaced, is caused by changes in the interference state that are induced by a spatially varying phase delay imparted on the probe beam by the plasma. This phase delay can be extracted from the image (Swadling et al. 2014) and is proportional to the electron column density of the plasma, $n_{\mathrm{e}} L$ (in $\mathrm{cm}^{-2}$ ). Here $L$ is the length of plasma along the probing beam, which changes as a function of position in the plane of the image. The sufficiently good axial symmetry of the object allows applying Abel inversion (Hutchinson 2005) to the electron column density, resulting in the axisymmetric (radial) distribution of electron density $n_{\mathrm{e}}(r)$ (in $\mathrm{cm}^{-3}$ ) shown in Figure 3(b). The highest electron density near the axis, reaching $n_{\mathrm{e}} \sim 10^{19} \mathrm{~cm}^{-3}$ (up to $75 \%$ uncertainty due to errors in the choice of the central axis and left-right asymmetries), is observed in the narrow, compressed region formed above the bow shock (the postshock region), while the electron density in the flow below the bow shock (the preshock region) is $n_{\mathrm{e}} \sim(3 \pm 2) \times 10^{18} \mathrm{~cm}^{-3}$ (i.e., $\sim 60 \%$ uncertainty). We note that, although these errors seem large, for measurements of electron density off-axis these errors decrease rapidly and can reach values $\lesssim 20 \%$. The higher electron density present in the jet driving the bow shock is qualitatively consistent with the density contrast (heavy jet propagating through ambient medium) inferred from application of the one-dimensional momentum flux conservation argument introduced in the previous section.

The laser shadowgraphy diagnostic in Figure 3(c) shows several interesting features. First, there are two dark regions in the top part of the image positioned on both sides of the axis, and just below them a dark vertical region on axis. These features are produced by large density gradients, and their shape is consistent with compression of the jet to a diameter smaller than the initial jet diameter due to converging flows driven by large postshock pressures. Formation of such shocks was observed in numerical simulations of high Mach number jets propagating through ambient media (e.g., Norman et al. 1982), and the structure we see in the experiment is 


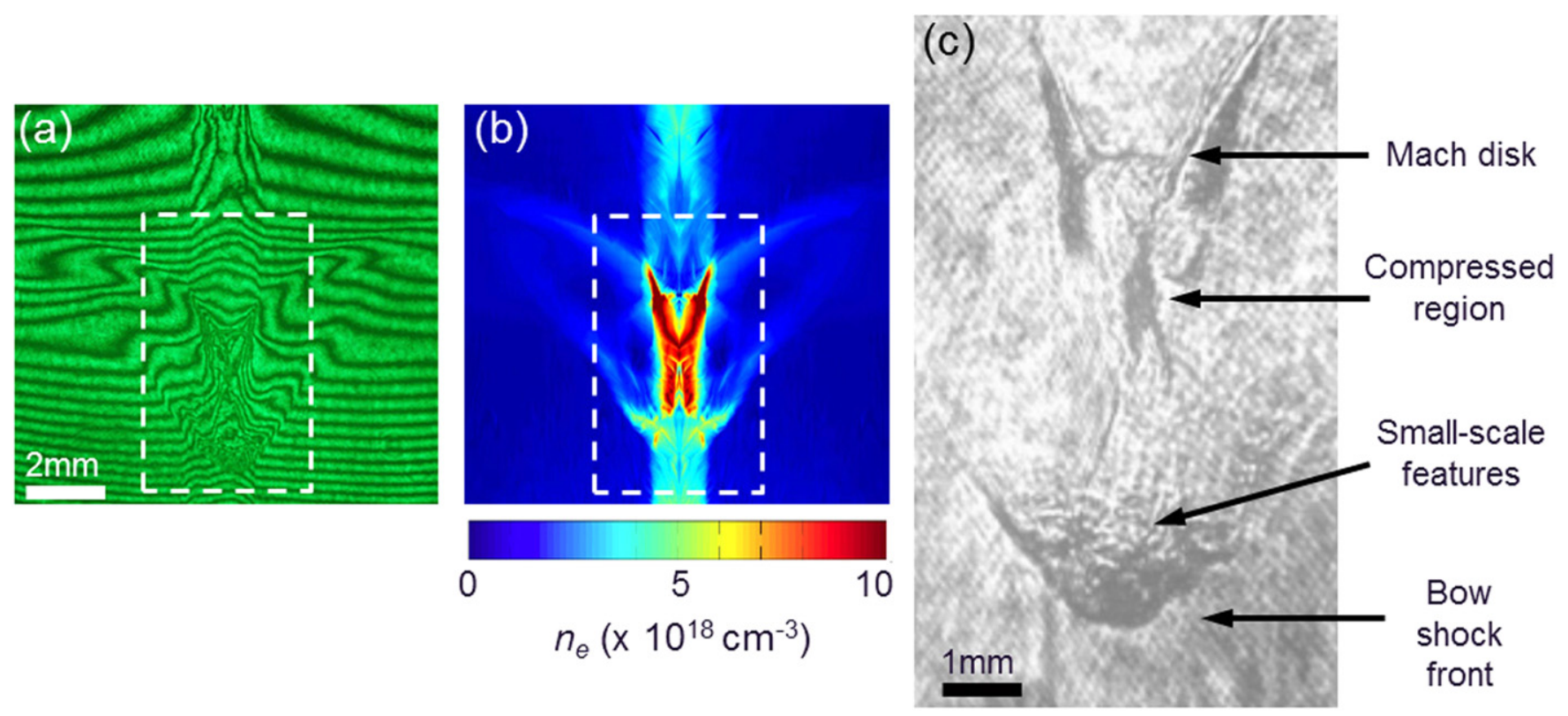

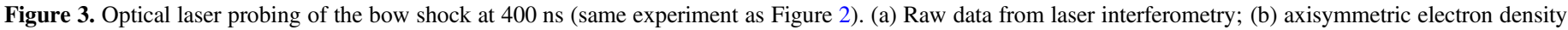

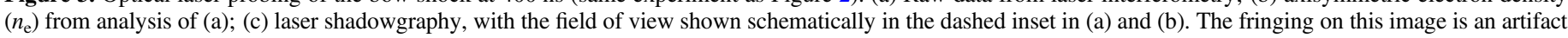
present in this particular diagnostic.

very similar to that observed in simulations of radiatively cooled jets with a similar density contrast, e.g., presented in Figure 7(c) of Blondin et al. (1990) for a density contrast of $\eta=3$, which is of the same order to the one inferred for these experiments of $\eta=6$. The simulations by Blondin et al. (1990) also show the presence of a second standing conical shock, positioned between the bow shock and the region of maximum compression that opens up toward the bow shock. The image in Figure 3(c) indicates that this conical shock is also present in the experiment, though it is less pronounced, indicating a smaller density gradient than that in the downstream conical shock. We also note the presence of a horizontal dark region connecting the dark features at the top of the image, which is consistent with the expected shape of a Mach disk (see, e.g., Figure 1(a) in Hartigan 1989 and Figure 7(b) in Blondin et al. 1990).

Finally, the image in Figure 3(c) shows the presence of small-scale structures already developing behind the bow shock at this early stage of the evolution. The perturbations appear to be elongated, predominantly in the direction normal to the jet axis, and the smallest detectable spatial scale is $\sim 120-170 \mu \mathrm{m}$, which is a factor of $\sim 3$ larger than the spatial resolution of the diagnostic $(\sim 50 \mu \mathrm{m}$ in the diffraction-limited case). We interpret the observed development of small-scale perturbations in the bow shock leading to its complete fragmentation later in time as being a result of strong radiative cooling in the postshock plasma. The fragmentation of the bow shock and the development of dense clumps in this region are associated with a thermal instability (Field 1965), which is discussed thoroughly in the next section. The fragmentation of shocks in protostellar jets due to thermal instabilities has been previously studied by numerical simulations (see, e.g., Blondin et al. 1989, 1990; Frank et al. 1998); however, the effect of this instability in YSO jets and shocks is still unclear.

\subsection{Radiative Cooling and Thermal Instabilities in the Bow Shock}

The most striking result in the experiments is the rapid development of small-scale spatial structures in the bow shock and postshock region. Our interpretation is that the fragmentation of the bow shock is related to a dynamic, local thermal instability that leads to the condensation of density perturbations by radiative cooling (see, e.g., Field 1965; Mathews \& Bregman 1978; Fall \& Rees 1985; Balbus 1986 and Blondin \& Cioffi 1989). The instability develops over the characteristic radiative cooling time, $\tau_{\text {cool }}$, which for optically thin plasmas, such as the ones in our experiments (Espinosa et al. 2015), is given by the ratio of thermal energy density, $U$, to the radiated power per unit volume $P_{\text {rad }}=n_{\mathrm{e}} n_{\mathrm{i}} \Lambda\left(n_{\mathrm{i}}, T_{\mathrm{e}}\right)$ as $\tau_{\text {cool }}=U / n_{\mathrm{e}} n_{\mathrm{i}} \Lambda$ $\left(n_{\mathrm{i}}, T_{\mathrm{e}}\right)$, where $\Lambda\left(n_{\mathrm{i}}, T_{\mathrm{e}}\right)$ is the normalized cooling function (in $\mathrm{erg} \mathrm{cm} \mathrm{cm}^{3}$ ) and $n_{\mathrm{i}}$ is the ion density. This expression can also be written as (Ryutov et al. 1999)

$$
\tau_{\text {cool }}[\mathrm{s}]=2.4 \times 10^{-12} \frac{(\bar{Z}+1) T[\mathrm{eV}]}{\bar{Z} n_{\mathrm{i}}\left[\mathrm{cm}^{-3}\right] \Lambda\left(n_{\mathrm{i}}, T_{\mathrm{e}}\right)},
$$

where $\bar{Z}$ is the average ionization in the plasma.

When radiative cooling occurs on timescales that are long compared to the sound speed crossing time, perturbations over a region of size $\lambda_{\text {iso }}=c_{\mathrm{s}} \tau_{\text {cool }}$ (where $c_{\mathrm{s}}$ is the ion acoustic speed) tend to maintain a common pressure (isobaric). In this case $n T \sim$ constant and the cooling rate then scales as $\Gamma=\tau_{\text {cool }}^{-1} \propto \Lambda(T) / T^{2}$. In the isobaric regime, if the cooling rate increases for a decreasing temperature, i.e., $d \Gamma / d T<0$, then radiation losses will be even more efficient in removing energy from the plasma and further reducing its temperature. To maintain pressure equilibrium with its surroundings, the density increases, thus further increasing the radiated power losses and potentially leading to a runaway condensation of the initial density perturbations. Detailed analysis of the instability 

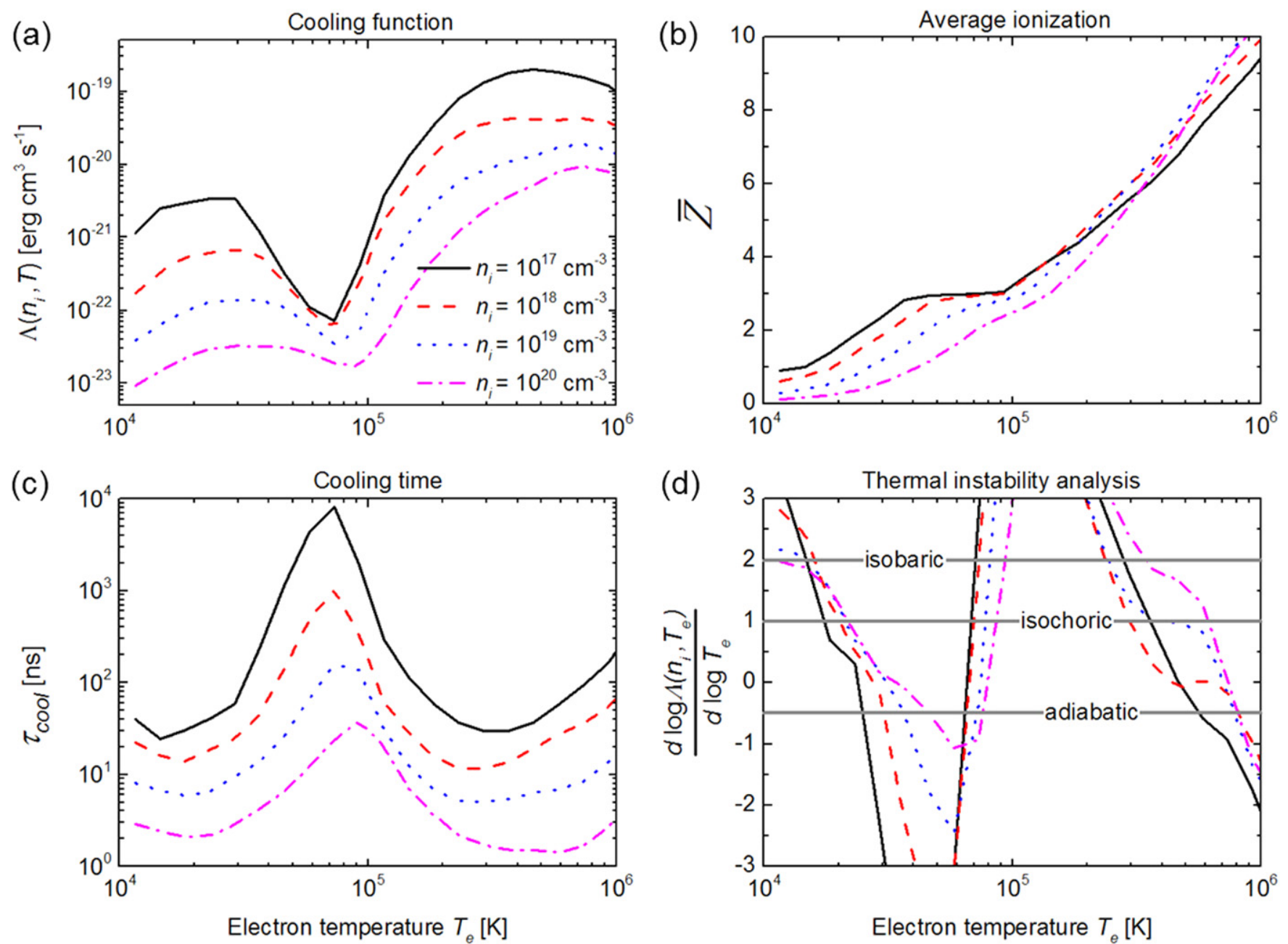

Figure 4. Calculated (a) cooling function and (b) average ionization for aluminum as a function of electron temperature for different ion densities relevant to our experiments (the legend shown in (a) applies to the entire figure). (c) Cooling time calculated using Equation (1). (d) Thermal instability analysis applied to the cooling functions shown in (a). The regions below each horizontal line indicate the thresholds for the onset of isobaric $(<2)$, isochoric $(<1)$, and adiabatic $(<-0.5)$ thermal instabilities.

was done by Field (1965), Hunter (1970), and Balbus (1986). For isobaric modes, the condition for stability is given by

$$
\frac{d\left(\log \Lambda\left(n_{\mathrm{i}}, T\right)\right)}{d(\log T)}<2 .
$$

While $\lambda_{\text {iso }}$ sets an upper limit for the the length scale of isobaric condensation, thermal conduction will suppress shortwavelength perturbations that are of the order of the so-called Field's length $\quad\left(\lambda_{\text {Field }}=2 \pi\left[(\gamma-1) \chi_{\text {th }} \tau_{\text {cool }}\right]^{1 / 2}\right)$, where $\chi_{\mathrm{th}}=\kappa / n_{\mathrm{e}}$ is the thermal diffusivity and $\gamma$ is the ratio of heat capacities. The most unstable wavelength, valid in the regime of large wavenumbers (but still smaller than a critical wavenumber $k_{\text {crit }}=2 \pi \lambda_{\text {Field }}^{-1}$ ), is given by the geometric mean (Field 1965) $\lambda_{\max }=\left(\lambda_{\text {Field }} \lambda_{\text {iso }}\right)^{1 / 2}$ so that density and temperature perturbations will be unstable in the wavelength range

$$
\lambda_{\text {Field }}<\lambda<\lambda_{\text {iso }} .
$$

Although cooling in our experiments is different from that in astrophysics owing to the differences in elements and physical conditions of temperature and densities, for optically thin plasmas the relevant parameters for comparison are the dimensionless cooling parameter $\chi_{\mathrm{cool}}$ and the dependence of the cooling function $\Lambda\left(n_{\mathrm{i}}, T\right)$ on temperature. To estimate the effects of radiative cooling in the postshock aluminum plasma, we use new cooling rates calculated with the computational packages ABAKO/RAPCAL (Rodriguez et al. 2008; Florido et al. 2009). The codes calculate the plasma level populations and average ionizations by solving the set of rate equations of the collisional-radiative model implemented in ABAKO (assuming that the plasma is optically thin and in steady state). The model is capable of accounting for coronal equilibrium and local and nonlocal thermodynamic equilibrium regimes. The atomic data required were obtained using the FAC code (Gu 2008) in the relativistic detailed configuration accounting approach, with the spin-orbit split array formalism (BaucheArnoult et al. 1985) and including configuration interaction within the same nonrelativistic atomic configurations. The databases of cooling functions and average ionizations obtained with ABAKO/RAPCAL were subsequently parameterized as a function of the plasma density and temperature using the PARPRA code (Rodriguez et al. 2014). Results from these numerical calculations are presented in Figures 4(a)-(b), showing the variation of the cooling function $\Lambda\left(n_{\mathrm{i}}, T_{\mathrm{e}}\right)$ and the average ionization $\bar{Z}$ for an aluminum plasma as a function of electron temperature (in the range of $\sim 10^{4}-10^{6} \mathrm{~K}$, 
$\sim 1-85 \mathrm{eV}$ ) for different ion densities typical of our experiments $\left(n_{\mathrm{i}}=10^{17}-10^{20} \mathrm{~cm}^{-3}\right)$. Figure 4(a) shows the rate of cooling increases with decreasing ion density, varying up to two orders of magnitude. The average ionization in Figure 4(b) shows an increase with temperature, with little variation as a function of ion density. With the cooling function and the average ionization we can calculate the cooling time $\tau_{\text {cool }}$ (Equation (1)), and this is plotted for different aluminum ion densities in Figure 4(c). The plot shows that overall the cooling time decreases with increasing ion density, as $\tau_{\text {cool }} \propto$ $1 / \Lambda\left(n_{\mathrm{i}}, T_{\mathrm{e}}\right)$, and longer cooling times can be expected around an electron temperature of $T_{\mathrm{e}} \sim 10^{5} \mathrm{~K}$. With the cooling time we can calculate the cooling parameter $\chi_{\text {cool }}=\tau_{\text {cool }} / \tau_{\text {hydro }}$, which quantifies the importance of radiative losses in the plasma. Taking the estimated hydrodynamical time of the experiments of $\tau_{\exp } \sim 10 \mathrm{~ns}$ (see Section 2.1), we see that, independent of ion density, $\tau_{\text {cool }}>\tau_{\exp }$ in the region around $T_{\mathrm{e}} \sim 10^{5} \mathrm{~K}$, which implies $\chi_{\text {cool }} \gtrsim 1$ and thus that radiative losses are not important. This region correlates with a dip in the cooling functions at this temperature. Besides this temperature region, however, the cooling time is overall of the order of the hydrodynamical time, and thus the cooling parameter $\chi_{\text {cool }} \sim 1$. The hydrodynamical time can also be estimated as the time it takes for the shock to form and become fully disrupted, which from Figure 2 can be taken as $\tau_{\exp } \sim 550-370 \mathrm{~ns}=180 \mathrm{~ns}$, resulting in $\chi_{\text {cool }} \sim(3-30 \mathrm{~ns}) / 180 \mathrm{~ns}<1$, and thus the plasma in the shock is expected to be radiatively cooled.

Knowledge of the dependence of the cooling function as a function of temperature allows performing a thermal instability analysis. The stability condition for isobaric modes presented in Equation (2) $\left(d \log \Lambda\left(n_{\mathrm{i}}, \quad T_{\mathrm{e}}\right) / d \log T_{\mathrm{e}}<2\right)$ is plotted in Figure 4(d) using the cooling functions for different ion densities in Figure 4(a). The threshold for isobaric instabilities shows that, almost independently of the ion density, the plasma is expected to become unstable at temperatures of $T_{\mathrm{e}} \sim(1.5-9) \times 10^{4} \mathrm{~K} \quad(\sim 5 \mathrm{eV})$ and at $T_{\mathrm{e}} \gtrsim 2.5 \times 10^{5} \mathrm{~K}$ $(\gtrsim 20 \mathrm{eV})$. Following the analysis in Shchekinov (1978), we also plot other instability thresholds, namely, isochoric and adiabatic modes, which correspond to $d \log \Lambda\left(n_{\mathrm{i}}, T_{\mathrm{e}}\right) / d \log T_{\mathrm{e}}<1$ and $<-0.5$, respectively.

Furthermore, the onset of thermal instabilities in the postshock plasma depends on the thermal evolution of the ion and electron plasma components. Because of the large difference between the ion and electron masses, there is a large temperature difference between the ion and electron components immediately behind the bow shock. In the strong-shock approximation (Mach number $M \gg 1)$, the postshock ion temperature is $T_{\mathrm{i}, \mathrm{PS}}=2(\gamma-1) A m_{\mathrm{p}} V_{\text {bow rel }}^{2} / k_{B}(\gamma+1)^{2} \sim 10^{6} \mathrm{~K}$, where $A$ is the atomic weight $\left(A=27\right.$ for aluminum), $m_{\mathrm{p}}$ is the proton mass, $V_{\text {bow rel }}$ is the shock velocity in the reference frame of a stationary preshock medium (i.e., $V_{\text {bow rel }}=100 \mathrm{~km} \mathrm{~s}^{-1}$ ), and $\gamma=5 / 3$ assuming an ideal gas. Compared to the ions, the electrons are compressed adiabatically across the shock and their temperature increases only by a factor of $4^{\gamma-1} \sim 2.5$ to $T_{\mathrm{e}}$, PS $\sim(2-3) \times 10^{5} \mathrm{~K}$. Although the plasma can be unstable at these temperatures, the existence of a well-defined unstable range of wavelengths, as given in Equation (3), is not satisfied. The postshock is characterized by a relaxation region where the energy exchange between ions and electrons competes with radiative cooling. Immediately after the shock, the electrons are thermally decoupled from the ions and their cooling timescale $(\lesssim 1 \mathrm{~ns})$ is much shorter than the ion-electron energy equilibration timescale $\left(\tau_{\epsilon}^{\mathrm{i} / \mathrm{e}}=\tau_{\epsilon}^{\mathrm{e} / \mathrm{i}} / \bar{Z} \sim 20 \mathrm{~ns}\right)$. Thus, over timescales of a nanosecond, the electron temperature rapidly drops while the ion temperature remains essentially constant. The cooling rate also decreases considerably, and an equilibrium is immediately reached behind the shock, where electron heating due to the energy received by the ions balances their radiative energy losses

$$
\frac{T_{\mathrm{i}}-T_{\mathrm{e}}}{\tau_{\epsilon}^{\mathrm{e} / \mathrm{i}}} \approx(\gamma-1) \frac{n_{\mathrm{i}}}{k_{B}} \Lambda\left(n_{\mathrm{i}}, T_{\mathrm{e}}\right) .
$$

In this regime $\tau_{\epsilon}^{\mathrm{i} / \mathrm{e}} \approx \tau_{\text {cool }} \sim 15-20 \mathrm{~ns}$ and the electrons are approximately isothermal. Detailed calculations of temperature equilibration show that the equilibrium electron temperature in the postshock relaxation layer is $T_{\mathrm{e}} \sim(1-1.5) \times 10^{5} \mathrm{~K}$; thus, the plasma is expected to be thermally stable. After $\sim 40 \mathrm{~ns}$ the electron and ion plasma components are fully equilibrated and their common temperature decreases below $\lesssim 7 \times 10^{4} \mathrm{~K}$. This places the plasma in conditions corresponding to a thermally unstable regime. The condition on the wavelengths is satisfied, and density and temperature perturbations with wavelengths $\lambda \sim 30-100 \mu \mathrm{m}$ grow over very short timescales of the order of $\lesssim 1 \mathrm{~ns}$.

\section{CONCLUSIONS}

We presented a new experimental configuration that aims at studying experimentally the formation of bow shocks relevant to those present in young stellar jets. In our experiments we produce two counterpropagating, supersonic plasma jets from plasma ablation of two radial foil Z-pinches. A bow shock is driven by the head-on collision between the two jets, and the bow shock properties are determined by their relative velocities and densities. A key result is that initially the bow shock is smooth but then quickly, within timescales of $\sim 30 \mathrm{~ns}$, develops small-scale spatial features behind the shock front. This timescale is consistent with the expected cooling times in the experiments, which were estimated using the cooling functions and average ionization calculated with the radiative packages ABAKO/RAPCAL. This allowed performing a thermal instability analysis for isobaric modes, resulting in expected temperature ranges at which the plasma should become thermally unstable. Detailed analytical calculations of the thermal evolution of the ion and electron components predict, for the experimental shock conditions, the development of an isobaric thermal instability in timescales of $\sim 40 \mathrm{~ns}$ and with typical spatial scales of $\sim 30-100 \mu \mathrm{m}$. This is in very good agreement with our experimental results that show an initially smooth bow shock that, within $\sim 30 \mathrm{~ns}$, fragments into smallscale spatial features with typical sizes of $\sim 120 \mu \mathrm{m}$.

Thermal instabilities may play a role in $\mathrm{HH}$ shocks provided that the shock velocities are high enough to raise the temperature in the postshock gas significantly above the peak of the cooling curve around $2 \times 10^{5} \mathrm{~K}$. The critical shock velocity for the onset of cooling instabilities has been estimated to be $\sim 200 \mathrm{~km} \mathrm{~s}^{-1}$ in 1D simulations (Smith 1989), though Sutherland et al. (2003) found that thermal instabilities in their 2D simulations generated filaments and voids in the postshock gas for shock velocities as low as $120 \mathrm{~km} \mathrm{~s}^{-1}$. However, Innes (1992) demonstrated that even a fairly weak preshock magnetic field stabilized shocks up to $175 \mathrm{~km} \mathrm{~s}^{-1}$. In $\mathrm{HH}$ jets, most shocks have velocities $\lesssim 100 \mathrm{~km} \mathrm{~s}^{-1}$ and should not be affected by thermal instabilities. However, the strongest bow shocks, such as $\mathrm{HH} 1$ and $\mathrm{HH}$ 2, have high ionization lines (Boehm 


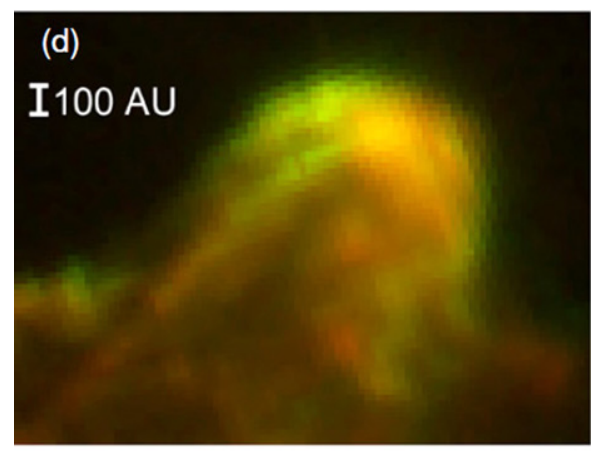

1994.6 UT

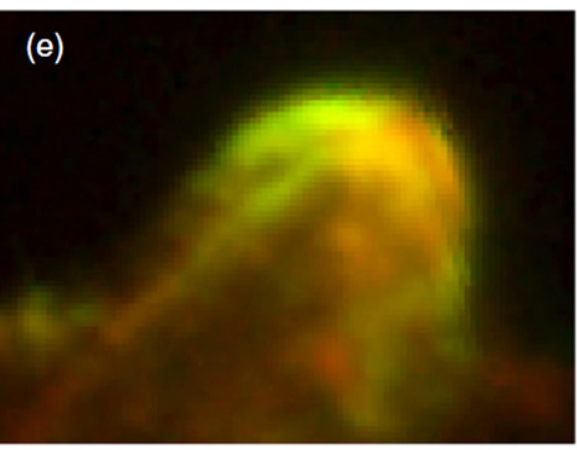

1997.6 UT

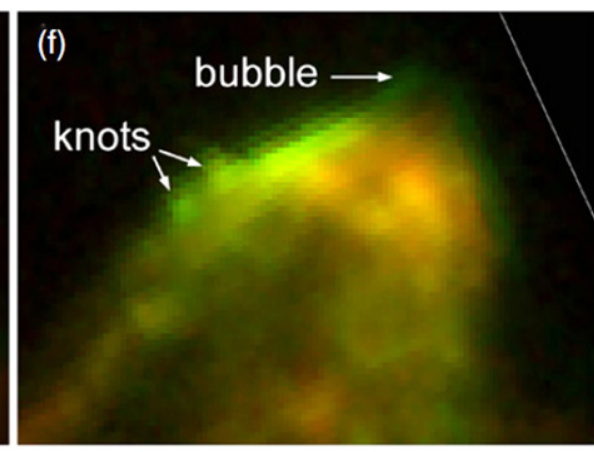

2007.6 UT

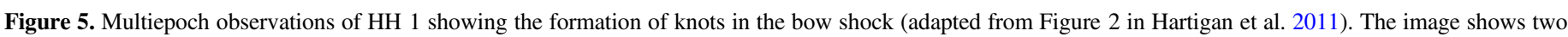
out of three epochs of HST images, with $\mathrm{H} \alpha$ in green, [S II] in red, and yellow denoting emission in both filters.

et al. 1993) and broad line profiles (Hartigan et al. 1987) indicative of shock velocities $\sim 200 \mathrm{~km} \mathrm{~s}^{-1}$, well above the criteria for thermal instabilities. As shown in Figure 5, bright knots do appear along the bow shock in HH 1 on timescales of decades. Using the scaling presented at the end of Section 2.1, the laboratory temporal scale of $30 \mathrm{~ns}$ would correspond to an astrophysical timescale of $\sim 17 \mathrm{yr}$, consistent with the astronomical observations. The spatial scales associated with the astronomical knots also scale well with the nonuniformities in the experiment. For example, the minimum size of the nonuniformities in the experiments of $0.12 \mathrm{~mm}$ scales to $4 \mathrm{AU}$ in the astronomical observations, where the spatial resolution is $\sim 20 \mathrm{AU}$. The larger nonuniformities present in the optical self-emission (e.g., the last three panels of Figure 2) have a typical size of $\sim 1 \mathrm{~mm}$, which scales to $30 \mathrm{AU}$ in Figure 5, consistent with the size of the new knots in HH 1. Driven from the same source on the other side of the outflow, HH 2 also has a high shock velocity and shows small knots that appear and merge along the strongest shock fronts (Hartigan et al. 2011). Of course, there are other ways to generate clumps along bow shocks, such as Kelvin-Helmholtz instabilities or even a clumpy preshock density, and we cannot rule out these possibilities without further observational data.

More generally, the thermal instability analysis could be applied to cooling curves used for numerical simulations of protostellar jets and shocks (see, e.g., Dalgarno \& McCray 1972; Kafatos 1973; Sutherland \& Dopita 1993). Although in these cases we expect the temperature ranges for the onset of thermal instabilities to differ from those expected in the experiments owing to the different elements and abundances that characterize them (e.g., $\mathrm{H}$ in simulations compared to $\mathrm{Al}$ in the experiments), in both cases the instability should develop at the appropriate slope of the cooling curve by the condition given in Equation (2).

The experiments presented here are important as they shed light on the onset and nonlinear evolution of the thermal instability. Numerical simulations of thermally unstable flows can be challenging as thermal conduction must be explicitly included to suppress the growth of small-scale perturbations. In particular, the Field's length has to be resolved by at least a few computational cells (Koyama \& Inutsuka 2004) to avoid the growth of perturbation and fragmentation at the grid scale. Furthermore, the presence of magnetic fields further increases the complexity of numerical calculations by making thermal conduction anisotropic. In that direction, similar experiments to the ones presented here can be designed to increase sufficiently the magnetic field in the flow to allow studying such a regime in the future. This could be relevant to previous theoretical studies that predict the suppression of thermal instabilities by magnetic fields (e.g., Innes 1992; Lesaffre et al. 2004).

This work was supported in part by the Royal Society through a University Research Fellowship, by EPSRC Grant No. EP/G001324/1, by DOE cooperative agreements No. DEF03-02NA00057 and No. DE-SC-0001063, and by the LABEX Plas@Par project. This work received financial state aid managed by the Agence Nationale de la Recherche, as part of the program "Investissements d'avenir" under ref. ANR-11IDEX-0004-02.

\section{REFERENCES}

Asahina, Y., Ogawa, T., Kawashima, T., et al. 2014, ApJ, 789, 79 Balbus, S. A. 1986, ApJL, 303, L79

Bauche-Arnoult, C., Bauche, J., \& Klapisch, M. 1985, PhRvA, 31, 2248

Blondin, J. M., \& Cioffi, D. F. 1989, ApJ, 345, 853

Blondin, J. M., Fryxell, B. A., \& Konigl, A. 1990, ApJ, 360, 370

Blondin, J. M., Konigl, A., \& Fryxell, B. A. 1989, ApJL, 337, L37 Boehm, K.-H., Noriega-Crespo, A., \& Solf, J. 1993, ApJ, 416, 647

Ciardi, A., Lebedev, S. V., Frank, A., et al. 2009, ApJL, 691, L147 Dalgarno, A., \& McCray, R. A. 1972, ARA\&A, 10, 375

de Gouveia Dal Pino, E. M. 2005, AdSpR, 35, 908

de Gouveia dal Pino, E. M., \& Benz, W. 1993, ApJ, 410, 686

de Gouveia dal Pino, E. M., \& Benz, W. 1994, ApJ, 435, 261

Drake, R. P., Davison, L., \& Horie, Y. 2006, High-Energy-Density Physics: Fundamentals, Inertial Fusion, and Experimental Astrophysics (Berlin: Springer)

Espinosa, G., Gil, J., Rodriguez, R., et al. 2015, HEDP, 17, 74

Falize, É., Michaut, C., \& Bouquet, S. 2011, ApJ, 730, 96

Fall, S. M., \& Rees, M. J. 1985, ApJ, 298, 18

Field, G. B. 1965, ApJ, 142, 531

Florido, R., Rodríguez, R., Gil, J. M., et al. 2009, PhRvE, 80, 056402

Frank, A., Ryu, D., Jones, T. W., \& Noriega-Crespo, A. 1998, ApJL, 494, L79

Frank, A., Ray, T. P., Cabrit, S., et al. 2014, Protostars and Planets VI (Tuscon, AZ: Univ. Arizona Press)

Gourdain, P.-A., Blesener, I. C., Greenly, J. B., et al. 2010, PhPl, 17, 012706 Gourdain, P.-A., \& Seyler, C. E. 2013, PhRvL, 110, 015002

Gu, M. F. 2008, CaJPh, 86, 675

Hartigan, P. 1989, ApJ, 339, 987

Hartigan, P. 2003, Ap\&SS, 287, 111

Hartigan, P., Foster, J. M., Wilde, B. H., et al. 2009, ApJ, 705, 1073

Hartigan, P., Frank, A., Foster, J. M., et al. 2011, ApJ, 736, 29

Hartigan, P., Morse, J. A., Reipurth, B., Heathcote, S., \& Bally, J. 2001, ApJL, 559, L157

Hartigan, P., Raymond, J., \& Hartmann, L. 1987, ApJ, 316, 323

Hunter, J. H., Jr. 1970, ApJ, 161, 451

Hutchinson, I. H. 2005, Principles of Plasma Diagnostics (Cambridge: Cambridge Univ. Press) 
Innes, D. E. 1992, A\&A, 256, 660

Kafatos, M. 1973, ApJ, 182, 433

Koyama, H., \& Inutsuka, S.-i 2004, ApJL, 602, L25

Lesaffre, P., Chièze, J.-P., Cabrit, S., \& Pineau des Forêts, G. 2004, A\&A, 427, 147

Mathews, W. G., \& Bregman, J. N. 1978, ApJ, 224, 308

Mitchell, I. H., Bayley, J. M., Chittenden, J. P., et al. 1996, RScI, 67, 1533

Nicolaï, P., Stenz, C., Kasperczuk, A., et al. 2008, PhPl, 15, 082701

Norman, M. L., Winkler, K.-H., \& Smarr, L. 1983, BAAS, 15, 962

Norman, M. L., Winkler, K.-H. A., Smarr, L., \& Smith, M. D. 1982, A\&A, 113,285

Reipurth, B., Heathcote, S., Morse, J., Hartigan, P., \& Bally, J. 2002, AJ, 123,362

Remington, B. A., Drake, R. P., \& Ryutov, D. D. 2006, RvMP, 78, 755

Rodriguez, R., Espinosa, G., Gil, J., et al. 2014, CCoPh, 16, 612

Rodriguez, R., Florido, R., Gil, J., et al. 2008, LPB, 26, 433
Ryutov, D., Drake, R. P., Kane, J., et al. 1999, ApJ, 518, 821

Ryutov, D. D., Drake, R. P., \& Remington, B. A. 2000, ApJS, 127, 465

Ryutov, D. D., Remington, B. A., Robey, H. F., \& Drake, R. P. 2001, PhPl, 8, 1804

Shchekinov, I. A. 1978, SvA, 22, 182

Smith, M. D. 1989, MNRAS, 238, 235

Stone, J. M., \& Norman, M. L. 1993, ApJ, 413, 198

Sutherland, R. S., Bicknell, G. V., \& Dopita, M. A. 2003, ApJ, 591, 238

Sutherland, R. S., \& Dopita, M. A. 1993, ApJS, 88, 253

Suzuki-Vidal, F., Bocchi, M., Lebedev, S. V., et al. 2012, PhPl, 19, 022708

Suzuki-Vidal, F., Lebedev, S. V., Ciardi, A., et al. 2009, Ap\&SS, 322, 19

Suzuki-Vidal, F., Lebedev, S., Pickworth, L. A., et al. 2014, in APS Meeting Abstracts, Session J05: HEDP Laboratory Physics (Ridge, NY: APS), 00005

Swadling, G. F., Lebedev, S. V., Hall, G. N., et al. 2014, RScI, 85, 110000

Teşileanu, O., Mignone, A., Massaglia, S., et al. 2008, A\&A, 488, 429 